\title{
HYPERBOLIC SURFACES AND QUADRATIC EQUATIONS IN GROUPS
}

\author{
ZHI-BIN GU
}

(Communicated by Warren J. Wong)

\begin{abstract}
A group of a hyperbolic 2-complex $K$ is a group with its associated van Kampen diagrams satisfying a hyperbolic curvature condition and a link condition on the degree of the interior vertices. A solution of an equation $W\left(y_{1}, \ldots, y_{n}\right)=1$ in $K$, where $W$ is a path in a 2-complex $B$, is a mapping $\zeta: B \rightarrow K$ such that $\zeta \boldsymbol{W}=W\left(\zeta y_{1}, \ldots, \zeta y_{n}\right)$ is contractible in $K$. This solution $\zeta$ is free if there is a mapping $h: B \rightarrow K^{(1)}$ such that $W\left(h y_{1}, \ldots, h y_{n}\right)$ is contractible in $K^{(1)}$ and such that $\zeta=\pi h$, where $\pi$ is the projection $\pi: K^{(1)} \rightarrow K$. Our main result is that each quadratic equation $W=1$ has only finitely many nonfree solutions in $K$. Our tool is essentially the cancellation diagrams on surfaces developed by the present author based on work of Schupp.
\end{abstract}

\section{INTRODUCTION}

We will study solutions of quadratic equations in groups. Classification of solutions of such equations in terms of free and nonfree solutions is first studied by Schupp [10]. There he proves that all solutions of a quadratic equation of nonpositive Euler characteristic are free in groups satisfying certain irregular small cancellation conditions. Consequently the present author [5] improves Schupp's methods and extends his results to broader classes of groups, i.e., small cancellation quotients of free products, of amalgamated products, and of HNN-extensions. Here we shall study another class of groups, the groups of hyperbolic 2-complexes. Such groups have recently been studied by Gersten [3] and Pride [8].

A group of a hyperbolic 2-complex is, roughly speaking, a group $G$ that satisfies irregular small cancellation conditions $C(p)$ and $T(q)$ with $(1 / p+$ $1 / q)<1 / 2$. For example, the $C(3)$ and $T(7)$, or the $C(4)$ and $T(5)$, or the $C(6)$ and $T(4)$ groups are hyperbolic. For a clear discussion on the connection between small cancellation groups and groups of hyperbolic 2-complexes, we refer to Pride [8].

Received by the editors August 30, 1988 .

1980 Mathematics Subject Classification (1985 Revision). Primary 20F05; Secondary 20 F06.

Key words and phrases. Quadratic relations, cancellation diagrams, groups of hyperbolic 2complexes, free and nonfree solutions, hyperbolic surfaces. 
The main tool we will make use of is the modified van Kampen diagrams together with their geometric duals, the modified pictures, which are both developed in [5], i.e., the cancellation diagrams that are bounded by nonreduced contractible paths. And here we will use them in the same manner as there.

\section{NOTATION AND DEFINITIONS}

Let $X$ be a 1-complex with the vertex set $V$ and the edge set $E$. Let $K=\langle X \mid R\rangle$ be a 2-complex where $R$ is the set of defining paths. We shall always assume that the defining paths are cyclically reduced. Also, $R$ is symmetrized if all cyclic permutations of $r^{ \pm 1} \in R$ also belong to $R$. In case $X$ is a bouquet with a single vertex, we call $K$ a presentation. We shall always use $K=\langle X \mid R\rangle$ to denote a presentation. Also, we shall denote the 1-skeleton of $K$ by $K^{(1)}$.

Let $K, L$ be two presentations. A mapping of 2-complexes from $K$ to $L$ consists of a mapping of 1-complexes from $K^{(1)}$ to $L^{(1)}$ that takes contractible paths in $K$ to contractible paths in $L$.

Next, we introduce another 1-complex, written as $K^{\mathrm{st}}$, associated to the 2complex $K$. We call $K^{\text {st }}$ the star complex of $K$, which is defined as follows.

(1) The vertex set of $K^{\text {st }}$ is the set $E$.

(2) The edge set is defined on $R$ : if $e_{1} e_{2}$ is a subpath in a path $r_{i}$ of $R$, then $K^{\text {st }}$ has an edge from $e_{1}$ to $e_{2}^{-1}$.

Now we are ready to introduce hyperbolic 2-complexes. First, we define a weight function $m$ to be a mapping from the edge set $E$ of $K$ into the real numbers: $m: E \rightarrow \mathbf{R}$, with $m\left(e^{-1}\right)=m(e)$ for all $e$ in $E$. If $\gamma=e_{1} e_{2} \cdots e_{n}$ is a defining path in $K$, then we define $m(\gamma)=\sum_{i=1}^{n} m\left(e_{i}\right)$. Then, the interesting situation to us is that when we have the 2-complex $K$ together with a weight function $m$ defined on $K^{\text {st }}$. We denote this situation by $(K, m)$. Then we define the weight function $m^{*}$ on $K^{\text {st }}$ as follows: If $\gamma=e_{1} e_{2} \cdots e_{n}$ is a defining path, then $m^{*}(\gamma)=m\left(\alpha_{e_{1} \wedge e_{2}}\right)+\left(\alpha_{e_{2} \wedge e_{3}}\right)+\cdots+m\left(\alpha_{e_{n-1}} \wedge e_{n}\right)+m\left(\alpha_{e_{n}} \wedge e_{1}\right)$, where $\alpha_{e_{i} \wedge e_{i+1}}$ is the oriented edge of $K^{\text {st }}$ from $e_{i}$ to $e_{i+1}$ (see Figure 1).

Following Gersten, we call $(K, m)$ hyperbolic if the following hold:

(i) Link condition. If $s$ is a reduced nonempty closed path in $K^{\text {st }}$, then $m^{*}(s) \geq 2$.

(ii) Curvature condition. For each $r \in R, m^{*}(r)<L(r)-2$, where $L(r)$ is the length of $r$.

These conditions, as will be seen in $\S 3$, have clear geometric meanings on cancellation diagrams.

In passing, we mention that, in obtaining a combinatorial proof of RiemannHurwitz formula, Lyndon [6] used conditions similar to the above.

We now consider another presentation. Let $B=\langle Y \mid W\rangle$ be a presentation where $Y$ is a 1-complex (disjoint from $X$ ) and $W$ is the only defining path. Then $W$ is called quadratic if each edge of $Y$ occurring in $W$ occurs exactly twice in $W$. In general, a quadratic path $W$ defines a closed surface $S$ as the 


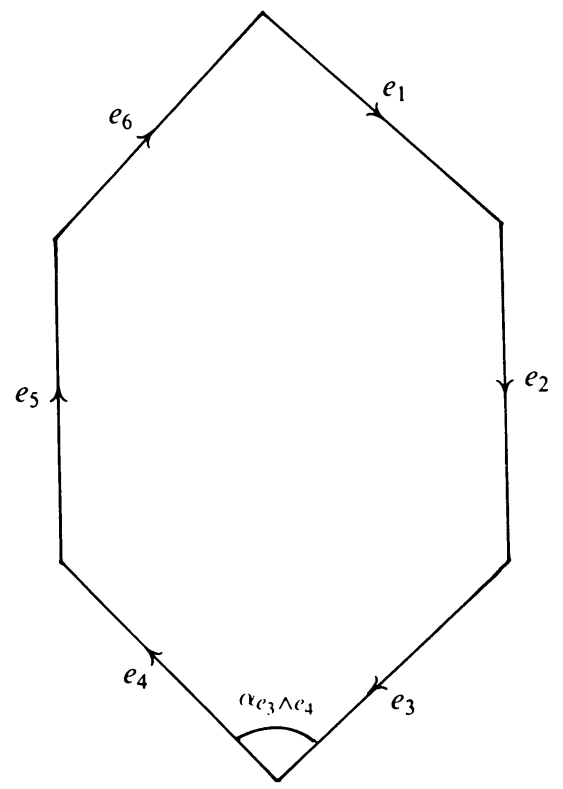

FiguRE 1.

quotient space of a polygonal region whose sides are labelled, in counterclockwise order, by the edges of $W$, with each pair of directed sides bearing the same label identified. This fact plays a crucial role in our work.

We say that $W$ is orientable (nonorientable) if the surface $S$ defined by $W$ is orientable (nonorientable). Then $W$ is orientable only if every edge of $Y$ occurring in $W$ occurs exactly twice, with opposite signs. Otherwise $W$ is nonorientable. We define the Euler characteristic of $E$ to be $\chi(W)=V-E+F$, the Euler characteristic of the surface defined by $W$, where $V, E$ and $F$ are the number of vertices, edges and regions of the surface.

Definition 2.1. A solution of $W\left(y_{1}, \ldots, y_{n}\right)=1$ in $K$ is a mapping $\zeta: B \rightarrow K$ such that $\zeta W=\left(\zeta y_{1}, \ldots, \zeta y_{n}\right)$ is contractible in $K$.

Definition 2.2. This solution $\zeta$ is free if there is a mapping $h: B \rightarrow K^{(1)}$ such that $W\left(h y_{1}, \ldots, h y_{n}\right)$ is contractible in $K^{(1)}$ and such that $\zeta=\pi h$, where $\pi$ is the projection $\pi: K^{(1)} \rightarrow K$.

The following are our main theorems, which we view as a supplement to the results for small cancellation groups [5].

Theorem B. Let $W\left(y_{1}, \ldots, y_{n}\right)$ be a quadratic path with $\chi(W) \geq 0$ in $B=$ $\langle Y \mid W\rangle$, and let $K=\langle X \mid R\rangle$ be hyperbolic where $R$ is symmetrized. Assume that $R$ contains no proper powers if $W$ is nonorientable. Then all solutions $\zeta W$ of the equation $W\left(y_{1}, \ldots, y_{n}\right)=1$ in $K$ are free. 
Theorem C. Let $W\left(y_{1}, \ldots, y_{n}\right)$ be a quadratic path with $\chi(W)<0$ in $B=$ $\langle Y \mid W\rangle$, and let $K=\langle X \mid R\rangle$ be hyperbolic where $R$ is symmetrized. Assume that $R$ contains no proper powers if $W$ is nonorientable. Then there exist only finitely many nonfree solutions of the equation $W\left(y_{1}, \ldots, y_{n}\right)$ in $K$.

\section{Cancellation diagrams}

Here we briefly state the definitions of a van Kampen diagram $M$ and its dual, the picture $P$. For more information, see [5].

Definition 3.1. A van Kampen diagram $M$ over a presentation $K=\langle X \mid R\rangle$ on a compact surface $S$ is a tessellation $M$ of $S$ with each region except one in $M$ having its boundary one of the defining paths of $R$, and the boundary path $W$ of the exceptional region $D_{\infty}$ is contractible in $K$.

Definition 3.2. Call $M$ a modified van Kampen diagram, written as $M^{*}$, if we don't require that $W$ be reduced.

We say that $M$ is not reduced if $M$ contains two regions $D_{1}$ and $D_{2}$ (not necessarily distinct) such that $\alpha$ is a common path of $D_{1} \cup D_{2}$ and the boundary path of $\left\{\partial D_{1} \cup \partial D_{2}-\alpha\right\}$ is a trivial path in $K$ (see Figure 2). To reduce $M$, we press together $D_{1}$ and $D_{2}$.

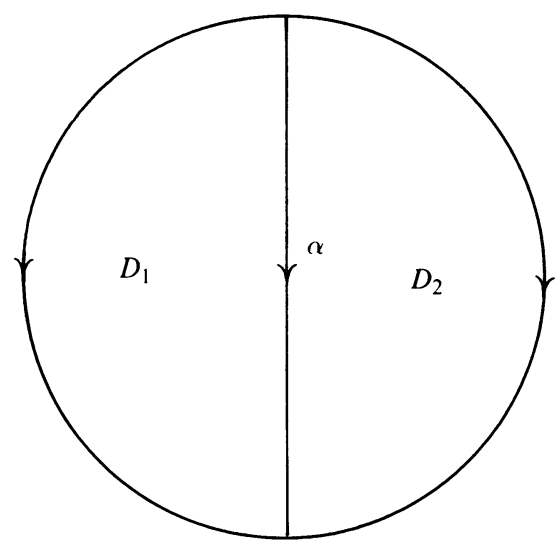

FIGURE 2.

Proposition 3.1 [5, Proposition 2.1]. A modified van Kampen diagram $M^{*}$ is obtainable from a reduced van Kampen diagram $M$ by attaching trees inside $D_{\infty}$ to the boundary $\partial D_{\infty}$.

Outline of proof. Let $W\left(x_{1}, \ldots, x_{n}\right)$ be a contractible path in $K$. Let $M_{0}$ be the "bouquet of balloons" given by $W\left(x_{1}, \ldots, x_{n}\right)=\prod_{i=1}^{n} u_{i} r_{i} u_{i}^{-1}$, and let the unreduced path $W_{0}$ be the boundary label of $M_{0}$. Let $W_{1}=x_{i_{1}} x_{i_{2}} \cdots x_{i_{m}}$ be the reduced form of $W_{0}$. Then this $W_{1}$ bounds a reduced van Kampen diagram $M_{1}$. We can pass from $W_{1}$ to $W_{0}$ by a succession of insertions of parts $x x^{-1}$. We modify $M_{1}$ to $M_{0}$ with boundary label $W_{1}$ by attaching, 
exterior to $M_{1}$, trees with each spur labelled $x x^{-1}$, i.e., to pass from $a b$ to $a x x^{-1} b$ (see Figure 3).

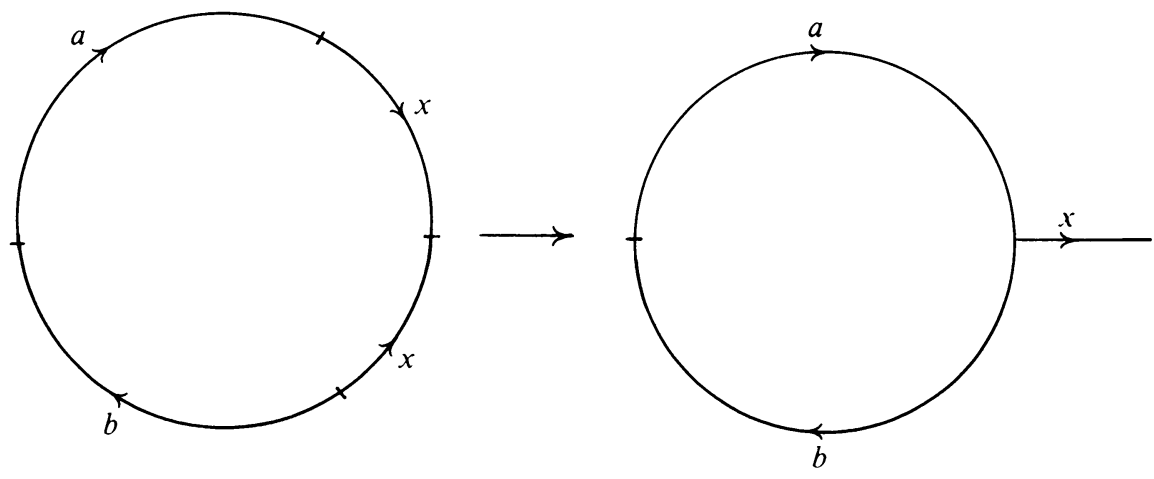

FIGURE 3.

Next, we consider the duals of $M$. We define a picture $P$ over $K=\langle X \mid R\rangle$ to be the geometric dual of a van Kampen diagram $M$ over $K$. Also a modified picture $P^{*}$ is the geometric dual of a corresponding modified van Kampen diagram $M^{*}$.

A picture $P$ is not reduced if $P$ contains two small discs $v_{1}^{*}$ and $v_{2}^{*}$ such that the paths around the arcs on $v_{1}^{*}$ and $v_{2}^{*}$ beginning at the end points of a common arc $e^{*}$ are mutually inverse paths in $K$ (see Figure 4 ).
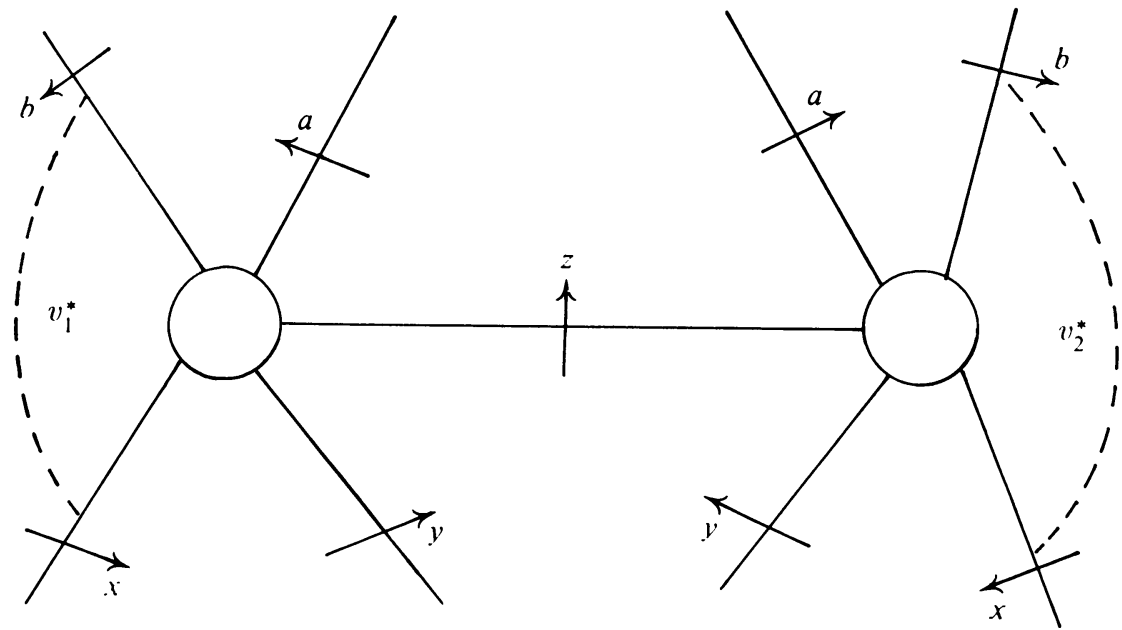

FIGURE 4.

To reduce $P$, we will use the so called bridge moves. A bridge move is the following move between two arcs with the same label but oppositely oriented (see Figure 5). 

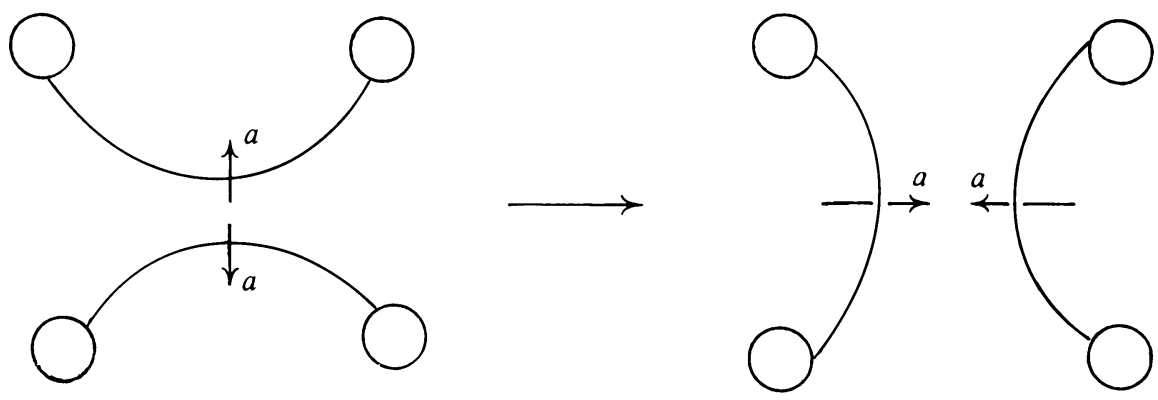

FIGURE 5.

Now on a cancellation diagram $M$, the meanings of the link and curvature conditions are clear. We see that $m^{*}(r)$ is the sum of the weights on interior corners of a region $D$ with boundary path $r$. We state these conditions on $M$ as follows.

(i) Link condition. For each reduced nonempty closed path $s$ in the star complex of the vertices of $M$, we have $m^{*}(s) \geq 2$.

(ii) Curvature condition. For each region $D$ of $M, m^{*}(D)<L(D)-2$, where $L(D)$ is the number of boundary edges of $D$.

Now on a picture $P$, these two conditions reverse.

(i) ${ }^{*}$ Link condition. For a small disc $v^{*}$ of $P$ with $\operatorname{arcs} e_{1}^{*}, \ldots, e_{n}^{*}$ attached, we have $m^{*}\left(v^{*}\right)<L\left(v^{*}\right)-2$, where $m^{*}\left(v^{*}\right)$ is the sum of the weights on oriented corners between $e_{i}^{*}$ and $e_{i+1}^{*}$ on $v^{*}$, and $L\left(v^{*}\right)$ is the number of arcs attached on $v^{*}$.

(ii) ${ }^{*}$ Curvature condition. For each region $\Delta$ of $P, m^{*}(\Delta) \geq 2$.

Definition 3.3. A tessellated surface $S^{T}$ is called hyperbolic if $S^{T}$ satisfies the link and curvature conditions.

\section{Main Results}

First, we have the following theorem that establishes a relation between a nonfree solution and a reduced nonempty tessellated surface $S^{T}$.

Theorem A [5, Theorem 3.1]. Let $W\left(y_{1}, \ldots, y_{n}\right)$ be a quadratic path in $B=$ $\langle Y \mid W\rangle$, and let $K=\langle X \mid R\rangle$ where $R$ is symmetrized. Assume that $R$ contains no proper powers if $W$ is nonorientable. If $K$ admits a non-free solution $\zeta W$ of the equation $W\left(y_{1}, \ldots, y_{n}\right)=1$, then there exists a reduced nonempty tessellated surface $S^{T}$ defined by $\zeta W$.

Outline of proof. Since $K$ admits a nonfree solution $\zeta W$, by Proposition 3.1 we have a modified van Kampen diagram $M^{*}$ defined by $\zeta W$. Now we transform $M^{*}$ to its dual, the modified picture $P^{*}$. Then, to obtain a closed surface, we identify the pairing arcs underlying the boundary $\partial P^{*}$. Next, we carry 
out reductions on this surface, hence obtaining a reduced nonempty tessellated surface, still denoted by $S^{T}$.

Now, to lift this surface $S^{T}$ up to a hyperbolic surface, written as $S^{H}$, we assume on $S^{T}$ the following conditions.

(i) ${ }^{*}$ For each region $\Delta$ of $S^{T}, m^{*}(\Delta) \geq 2$.

(ii) ${ }^{*}$ For each vertex $v^{*}$ in $S^{T}, m^{*}\left(v^{*}\right)<L\left(v^{*}\right)-2$, where $L\left(v^{*}\right)$ is the number of arcs around $v^{*}$.

Next, we have a crucial lemma.

Lemma 4.1. On the hyperbolic surface $S^{H}$, we have

$$
V \leq-\chi(W) / \varepsilon
$$

where $V$ is the number of vertices of $S^{H}, \chi\left(S^{H}\right)$ is Euler characteristic of $S^{H}$, and $\varepsilon=\operatorname{Minimum}\left\{L\left(v^{*}\right)-m^{*}\left(v^{*}\right)-2\right\}$.

Proof. In the following, all the summations are all over the entire $S^{H}$.

First we have

$$
\begin{aligned}
\sum_{v^{*}} m^{*}\left(v^{*}\right) & \leq \sum_{v^{*}}\left[L\left(v^{*}\right)-(2+\varepsilon)\right] \\
& \leq 2 E-(2+\varepsilon) V
\end{aligned}
$$

where we count the number of arcs with multiplicity. Second, we have

$$
\sum_{\Delta} m^{*}(\Delta) \geq 2 F
$$

Thus, combining (1) and (2) we have

$$
2 F \leq 2 E-(2+\varepsilon) V
$$

that is,

$$
2 \varepsilon V \leq-2(V-E+F)=-2 \chi\left(S^{H}\right) .
$$

Hence

$$
V \leq-\chi\left(S^{H}\right) / \varepsilon .
$$

Since the process of reductions of cancellation diagrams may decrease the genus of a surface, we have $\chi(W) \leq \chi\left(S^{H}\right)$, hence $-\chi(W) \geq-\chi\left(S^{H}\right)$. Therefore, from (3) we have

$$
V \leq \chi(W) / \varepsilon .
$$

Now readily we have the following two theorems.

Theorem B. Let $W\left(y_{1}, \ldots, y_{n}\right)$ be a quadratic path with $\chi(W) \geq 0$ in $B=$ $\langle Y \mid W\rangle$, and let $K=\langle X \mid R\rangle$ be hyperbolic where $R$ is symmetrized. Assume that $R$ contains no proper powers if $W$ is nonorientable. Then all solutions $\zeta W$ of the equation $W\left(y_{1}, \ldots, y_{n}\right)=1$ in $K$ are free.

Proof. If $\chi(W) \geq 0$, then Lemma 4.1 assures that the number of vertices in $S^{H}$ is zero, hence no such surface $S^{H}$ can occur. Therefore, we conclude that all solutions are free. 
Theorem C. Let $W\left(y_{1}, \ldots, y_{n}\right)$ be a quadratic path with $\chi(W)<0$ in $B=$ $\langle Y \mid W\rangle$, and let $K=\langle X \mid R\rangle$ be hyperbolic where $R$ is symmetrized. Assume that $R$ contains no proper powers if $W$ is nonorientable. Then there exist only finitely many nonfree solutions of the equation $W\left(y_{1}, \ldots, y_{n}\right)=1$ in $K$.

Proof. If $\chi(W)<0$, then Lemma 4.1 implies that the number of vertices of $S^{H}$ is bounded. Then for each surface $S^{H}$ with a fixed number of vertices, there exist only finite number of triangulated 2-complexes $B$ (including different labellings). Hence there are only finitely many nonfree solutions.

\section{ACKNOWLEDGMENTS}

This work was initiated during my visit to the University of Glasgow in the summer of 1986. I am greatly indebted to Jim Howie and Steve Pride for much assistance. Furthermore, I am very grateful to Roger Lyndon for comments.

\section{REFERENCES}

1. L. P. Comerford, Jr., and C. C. Edmonds, Quadratic equations over free groups and free products, J. Algebra 68 (1981), 276-297.

2. M. Culler, Using surfaces to solve equations in groups. Topology 20 (1981), 133-145.

3. S. M. Gersten, Reducible diagrams and equations over groups, preprint.

4. R. Z. Goldstein and E. C. Turner, Solving quadratic equations in groups, preprint.

5. Z-B. Gu, Cancellation diagrams on surfaces and quadratic equations in groups, (to be submitted).

6. R. C. Lyndon, On the combinatorial Riemann-Hurwitz formula, in Convegni sui gruppi infiniti, Rome 1973. Academic Press, New York and London, 1976, 435-439.

7. R. C. Lyndon and P. E. Schupp, Combinatorial group theory, Ergebnisse der math. 89, SpringerVerlag, 1977.

8. S. J. Pride, Star complexes, (to appear in Glasgow J. Math.).

9. C. Rourke, Presentations and the trivial group, in Topology of Low-Dimensional Manifolds, Lecture Notes in Math. Vol. 722, Springer-Verlag, 1979, 134-143.

10. P. E. Schupp, Quadratic equations in groups, cancellation diagrams on compact surfaces and automorphisms of surface groups, in Word Problems II: The Oxford Book, Amsterdam, 1980, 347-371.

11. H. B. Short, Topological methods in group theory: the adjunction problem, Ph.D. Thesis, Warwick, 1981.

Department of Mathematics, North Texas State University, Denton, Texas 76203

Current address: Department of Mathematics and Statistics, Bowling Green State University, Bowling Green, Ohio 43403 